\title{
Probing Energy Landscapes of Cytochrome bof with Spectral Hole Burning: Effects of Deuterated Solvent and Detergent
}

\author{
Alexander Levenberg ${ }^{1}$, Golia Shafiei ${ }^{1}$, Maria A. Lujan ${ }^{2}$, Steven Giannacopoulos ${ }^{1}$, \\ Rafael Picorel ${ }^{2}$ and Valter Zazubovich ${ }^{1, *}$ \\ ${ }^{1}$ Department of Physics, Concordia University, 7141 Sherbrooke Str. West, Montreal, Quebec \\ H4B 1 R6 Canada; ${ }^{2}$ Estacion Experimental de Aula Dei (CSIC), Avda. Montañana 1005, 50059 \\ Zaragoza, Spain.
}

\begin{abstract}
In Non-Photochemical Spectral Hole Burning (NPHB) and hole recovery experiments cytochrome $b_{6} f$ protein exhibits behavior that is almost independent of the deuteration of the buffer / glycerol glassy matrix containing the protein, apart from some differences in heat dissipation. On the other hand, strong dependence of hole burning properties on sample preparation procedures was observed and attributed to a large increase of the electron-phonon coupling and shortening of the excited-state lifetime occurring when $n$-dodecyl $\beta$-D-maltoside (DM) is used as a detergent instead of n-octyl $\beta$-D-glucopiranoside (OGP). The data was analyzed assuming that the tunneling parameter distribution or barrier distribution probed by NPHB and encoded into the spectral holes contains contributions from two non-identical components with accidentally degenerate excited state $\lambda$-distributions. Both components likely reflect protein dynamics, although with some small probability one of them (with larger $m d^{2}$ ) may still represent the dynamics involving specifically the $-\mathrm{OH}$ groups of the water / glycerol solvent. Single proton tunneling in the water / glycerol solvent environment or in the protein can be safely excluded as the origin of observed NPHB and hole recovery dynamics. The intensity dependence of the hole growth kinetics in deuterated samples likely reflects differences in heat dissipation between protonated and deuterated samples. These differences are most probably due to the higher interface thermal resistivity between (still protonated) protein and deuterated water/ glycerol outside environment.
\end{abstract}

*Corresponding author, E-mail: valter.zazubovits@ concordia.ca 


\section{Introduction.}

Spectral hole burning has been widely used to probe energy landscapes and low-temperature dynamics in various glasses, polymers and proteins ${ }^{1-3}$. Information on energy landscapes can be obtained from observing the burning process (HGK, hole growth kinetics), the recovery of the spectral hole and its broadening at fixed temperature as well as recovery and broadening upon thermocycling ${ }^{2,4-7}$. Consistent analysis of the data obtained by these methods for the same pigment / host system allows for determining the shapes and the parameters of the distributions of the barriers on the protein energy landscape and of the tunneling parameter $\lambda$, as well as for making inferences about the nature of the entities whose structural rearrangement is responsible for non-photochemical spectral hole burning, NPHB. In the case of NPHB, the pigment molecule is not chemically photo-transformed; it is the pigment molecule's environment that experiences some (reversible) change triggered by optical excitation. The basics of NPHB are illustrated by Figure 1A that also contains the definitions of some relevant parameters. Similar double-well potentials of the Two-Level System (TLS) model ${ }^{1-3,8,9}$ are used for both ground and excited electronic states of the pigment molecule in protein environment ("system"). TLS is used instead of the actual energy landscape with the larger number of minima for the sake of simplicity. (Further justification of using TLS can be found in the Results section.) Persistent NPHB involves the higher of the hierarchal tiers ${ }^{10-13}$ of the energy landscape shown in the Figure 1A, while the lower-tier structures at the bottoms of the wells are mostly responsible for the broadening of the spectral hole. When the photon energy matches the transition energy of the pigment molecule in configuration 1, the system will go to the excited state and then some small conformational change in the protein may bring the system to configuration 2 . The system may make the transitions between two wells by tunneling through the potential barrier (dashed 
diagonal arrow) or by hopping over the barrier (solid bent arrow). It is generally believed that at temperatures below $10 \mathrm{~K}$ the transition occurs by means of tunneling ${ }^{14,15}$. At higher

temperatures both mechanisms may be at work. The potential barrier in the excited state must be significantly lower than the one in the ground state for persistent NPHB to be possible. The tunneling parameter $\lambda$ can be thought of as a composite "barrier strength" and is expressed (in Wentzel, Kramers and Brillouin, WKB, approximation) via the parameters of the TLS presented in Figure 1B:

$$
\lambda=\frac{d}{\hbar} \sqrt{2 m V}
$$

where $V$ is the height of the energy barrier, $d$ is the distance between two energy minima along the generalized coordinate, $m$ is the effective mass of the entity that rearranges during the transitions between two conformational states.

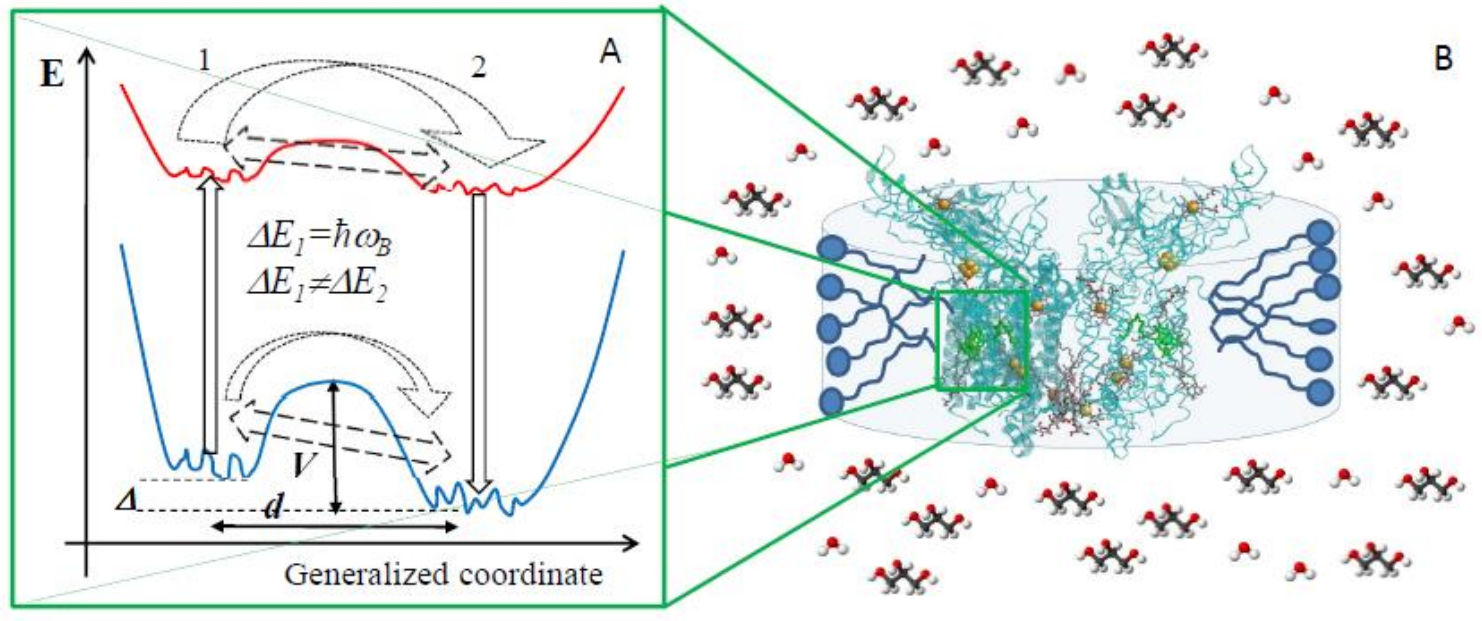

Figure 1: A: Overall view of the "system" containing a pigment molecule (with ground and excited electronic states) in interaction with the bi-stable local environment. See text for details. Fine structure at the bottom of the wells represents lower-barrier tier of the energy landscape. B: The assembly being explored in our experiments consists of the dimeric Cyt $\mathrm{b}_{6} \mathrm{f}$ protein solubilized with the help of either OGP or DM detergent and surrounded by buffer / glycerol 
mixture. The latter may be protonated or deuterated. Chlorophylls inside Cyt $b_{6} f$ protein are highlighted in green.

As stated above, $V$ and $\lambda$ are subject to distribution, while $m$ and $d$ are usually considered to be fixed. Also shown in Figure 1A is the asymmetry $\Delta$, i.e. the difference between the zero levels of the phonon/vibrational mode in two wells. The tunneling parameter $\lambda$ affects the NPHB quantum yield $\phi(\lambda)$, which is given by ${ }^{3}$

$$
\phi(\lambda)=\frac{\Omega_{0} \exp (-2 \lambda)}{\Omega_{0} \exp (-2 \lambda)+\tau_{1}^{-1}}
$$

where $\Omega_{0}$ is the attempt frequency, determining how often the systems try to pass the potential energy barrier between the two wells, and $\tau_{1}$ is the observable fluorescence lifetime, that may or may not be affected by energy transfer or other form of quenching, depending on the sample. After tunneling in the excited state the system returns to the ground state and gets trapped in configuration 2 . The parameters of the ground state determine how long it will take for the system to return to configuration 1.

Until recently, there were limited attempts at consistently interpreting different types of NPHB-related experiments as far as the shapes of the distributions were concerned. Hole growth kinetics (HGK) was modelled utilizing Gaussian distributions of the tunneling parameter $\lambda$ for more than 20 years ${ }^{3,16-21}$. Occasionally, Gaussian $\lambda$-distributions for the ground state of the chromophore-amorphous host system were employed in fitting hole recovery ${ }^{20}$ or inferred from the interplay between burning and simultaneous recovery at elevated temperatures ${ }^{21}$. On the other hand, hole recovery upon thermocycling was usually reported without giving much details about the preceding burning (such as fractional hole depth), and with few exceptions ${ }^{20}$ - without 
attempting to determine not only the barrier $(V)$ distributions, but also the $\lambda$-distributions. Modeling of thermocycling results often required utilizing non-Gaussian barrier distributions ${ }^{4-7}$. Barrier distributions proportional to $1 / \sqrt{V}$ yield rectangular $\lambda$-distributions (uniform between certain $\lambda_{\min }$ and $\lambda_{\max }$ ). Gaussian distributions of $\lambda$, on the other hand, should yield only slightly asymmetric (as long as the mean of the $\lambda$-distribution is much larger than its width) chi-squared type barrier height distributions, not resembling those derived from the thermocycling results ${ }^{4-7}$.

Lately, single molecule spectroscopy, SMS (or single pigment-protein complex spectroscopy, SPCS) has become the technique of choice for studying spectral dynamics resulting from the dynamics of the amorphous hosts, including proteins. However, it must always be questioned which of the low-temperature SMS or SPCS observations are light-induced (i.e. are single-molecule acts of NPHB), and which are occurring anyway and are merely monitored by optical methods ${ }^{19}$. Thus, NPHB that is utilizing much lower light intensities is still capable of offering many valuable insights. Next question is if barriers on the energy landscape are crossed via tunneling or thermally-activated hopping. SPCS experiments on LH2 complex suggested that both tunneling and barrier-hopping were responsible for the observed spectral dynamics ${ }^{22}$. Detailed understanding of light-induced small conformational changes in amorphous solids and proteins will be beneficial for interpreting both available and future SPCS results. Another interesting question pertaining specifically to pigment-protein complexes involved in light harvesting was raised in ${ }^{23}$ - does introduction of additional energy to the pigment-protein complex by exciting it via higher-energy pigments or excitonic states allow the environment of the lower-energy pigments to sample a larger set of conformational sub-states? Quantitative knowledge of the distributions of the barriers involved in NPHB and hole recovery will be 
critical for determining the degree of applicability of this idea to complexes featuring various donor-acceptor energy gaps.

The CP43 complex of spinach Photosystem II ${ }^{24}$ was the first where the same samples were subjected to measurements of HGK and both modes of hole recovery ${ }^{14}$. In this case, all observed barrier distributions appeared to be Gaussian, and there was no evidence for $\sim 1 / \sqrt{V}$ distributions predicted by the theories of amorphous solids and indeed apparently observed in glasses ${ }^{4,5}$ and some proteins ${ }^{6}$. On the other hand, in the case of cytochrome $(\mathrm{Cyt}) \mathrm{b}_{6} \mathrm{f}^{25}$ "glasslike" $\sim 1 / \sqrt{V}$ barrier distributions were observed ${ }^{15}$. This raised a question of intactness of our Cyt $b_{6} f$ samples. What if the chlorophylls (Chl) of our Cyt $b_{6} f$ sample were exposed to the buffer-glycerol mixture surrounding the protein (e.g. as a result of partial protein denaturation), and the observed spectral dynamics belonged to this glassy solid? The overall structure of the Cyt $b_{6} f$ sample is shown in Figure $1 \mathrm{~B}$ to facilitate understanding of this discussion. Although in ${ }^{15}$ we presented $\Delta$-FLN spectra indicating inter-pigment energy transfer with the rate following from the structural data ${ }^{25}$ and thus suggesting that our sample was intact, the possibility remained that the chlorophylls were still somehow reporting on the outside glass dynamics (either due to interactions with the outside TLS or surface TLS ${ }^{26}$ as proposed in ${ }^{2}$ or via protein "slaving" to the buffer-glycerol solvent dynamics ${ }^{27}$ ) and that true protein Cyt bof dynamics was not actually probed. Essentially, the question is - what elements of the sample structure depicted in Figure 1B give rise to the generalized coordinate in Figure 1A?

It is well known that spectral dynamics in many pigment-host systems is very sensitive to the exchange of protons to deuterons. (This is similar to a broader class of the phenomena where the rates of chemical reactions involving proton tunneling are strongly affected by deuteration.) In the cases when the pigment was in a direct contact with the deuterated hyperquenched 
amorphous water environment, the slowdown of NPHB by a factor of several hundred was observed $^{28}$, in agreement with the increase of the (mean of the distribution of) tunneling parameter $\lambda$ from 8 to 11.2 , i.e. by $\sqrt{2}$. Fifteen-fold decrease of the NPHB yield was observed in $\left[\mathrm{Cr}(\text { oxalate })_{3}\right]^{3-}$ in ethylene glycol/water ${ }^{29}$. On the other hand, a massive increase of NPHB yield upon partial deuteration in $\mathrm{NaMgAl}(\text { oxalate })_{3} \cdot 9 \mathrm{H}_{2} \mathrm{O}: \mathrm{Cr}(\mathrm{III})$ was attributed to opening an additional NPHB channel involving flipping of the DOH molecules ${ }^{30}$. Thus, here we will explore if deuterated environment affects the spectral dynamics of Cyt $\mathrm{b}_{6} \mathrm{f}$ as well as that of $\mathrm{Chl} a$ in buffer-glycerol protein-free environment. In the process of exploring $\mathrm{Cyt}_{\mathrm{b}} \mathrm{f}$ samples from different batches it also became evident that observed NPHB kinetics is sensitive to the choice of the detergent used in sample preparation. This effect is also going to be examined.

\section{Experimental.}

Two batches of dimeric Cyt $b_{6} f$ were used in this study. The first one was the same as employed in ${ }^{15}$; n-octyl $\beta$-D-glucopyranoside (OGP) was used to solubilize and stabilize the protein complex in solution. The other one utilized $n$-dodecyl $\beta$-D-maltoside, and was obtained as explained in ${ }^{31}$. Extinction coefficients of $25 \mathrm{mM}^{-1} \mathrm{~cm}^{-1}$ (at $\left.554 \mathrm{~nm}\right)^{32}, 21 \mathrm{mM}^{-1} \mathrm{~cm}^{-1}$ (at $563 \mathrm{~nm}$ ) ${ }^{32}$, and $75 \mathrm{mM}^{-1} \mathrm{~cm}^{-1}$ (at $\left.668.5 \mathrm{~nm}\right)^{33}$ were used for the Cyt f, Cyt b, and Chl, respectively. For experiments in deuterated environment both the water of the buffer and the glycerol (used as a cryoprotectant and to produce transparent glass at low temperatures) were substituted by at least 98\% deuterated compounds (i.e. $\mathrm{d}_{8}$-glycerol was used) purchased from C/D/N Isotopes. Pure Chl $a$ was purchased from Sigma-Aldrich. Samples were stored at $-80^{\circ} \mathrm{C}$ and unfrozen only immediately prior to the experiment. Thus, possible exchange of deuterons between solvent and protein or Chl $a$ as well as potential protein damage by glycerol were minimized. As Chl $a$ is 
very poorly soluble in water or glycerol, small amount of solid Chl $a$ was first dissolved in $<100$ $\mu \mathrm{L}$ of methanol, and then this concentrated solution was added to $\sim 1 \mathrm{~mL}$ of water-glycerol mixture immediately prior to the experiment. Deuterated methanol was purchased from $\mathrm{C} / \mathrm{D} / \mathrm{N}$ Isotopes as well.

Absorption spectra were measured either with a Cary 5000 spectrophotometer with resolution of $0.1 \mathrm{~nm}$ or with a home-built VIS-NIR spectrophotometer based on a Jobin-Yvon HR640 with bandwidth of about $0.04 \mathrm{~nm}$. Broadband emission spectra were measured using Acton 2356 spectrograph with a Princeton Instruments Pixis 1340x100 back-illuminated CCD. A $633 \mathrm{~nm}$ He-Ne laser was used for excitation. High-resolution spectra were measured with Sirah Matisse-DS frequency-stabilized tunable dye laser (bandwidth < 1 MHz). 6 W $532 \mathrm{~nm}$ Spectra Physics Millennia was used as a pump laser. The samples were placed in a 4 by $10 \mathrm{~mm}$ crosssection plastic cuvette surrounded by an aluminum mask inside a liquid helium bath / flow cryostat (Ukrainian Academy of Sciences) with temperature stabilized to better than $0.1 \mathrm{~K}$. Highresolution experiments were performed in fluorescence excitation mode, with vertically polarized excitation and with fluorescence detected with a Hamamatsu photomultiplier / photon counting module at $90^{\circ}$. The intensity of the excitation light was stabilized by BEOC laser power controller and regulated with absorptive neutral density filters. In experiments on Chl $a$ in solution to ensure that monomeric chlorophyll was preferentially probed in fluorescence excitation mode, fluorescence was collected in the $695-710 \mathrm{~nm}$ range only (Omega Optical 700AF20 +695AELP). For Cyt $b_{6} f$ the $700 \mathrm{~nm}$ long-pass (Omega Optical 700AELP) filter was used in front of the PMT. 


\section{Results.}

3.1. Deuterium effects: Figure 2A shows several HGK curves obtained at various temperatures from $5.5 \mathrm{~K}$ to $12.5 \mathrm{~K}$ in OGP sample in deuterated solvent. The curves follow the same general trends as were observed for $\mathrm{Cyt}_{6} \mathrm{~b}_{6}$ in protonated environment ${ }^{15}$ : (i) the hole deepening was slowing down with the increase of temperature, in agreement with the increase of the homogeneous line width and (ii) at elevated temperatures the initial several percent of burning were faster than expected, most likely due to the onset of burning via barrier-hopping in the excited state of the chlorophyll-protein system. Figure 2A also contains the 5.5 K HGK curve for protonated sample (grey noisy curve). Thus, at the first glance it seems that the tunneling parameter $\lambda$ increased by about $0.8(8 \%)$ in the deuterated sample and perhaps the $\lambda$-distribution width increased somewhat as well (parameters used to obtain smooth curves in frame A were fine-tuned somewhat with respect to Ref 15).
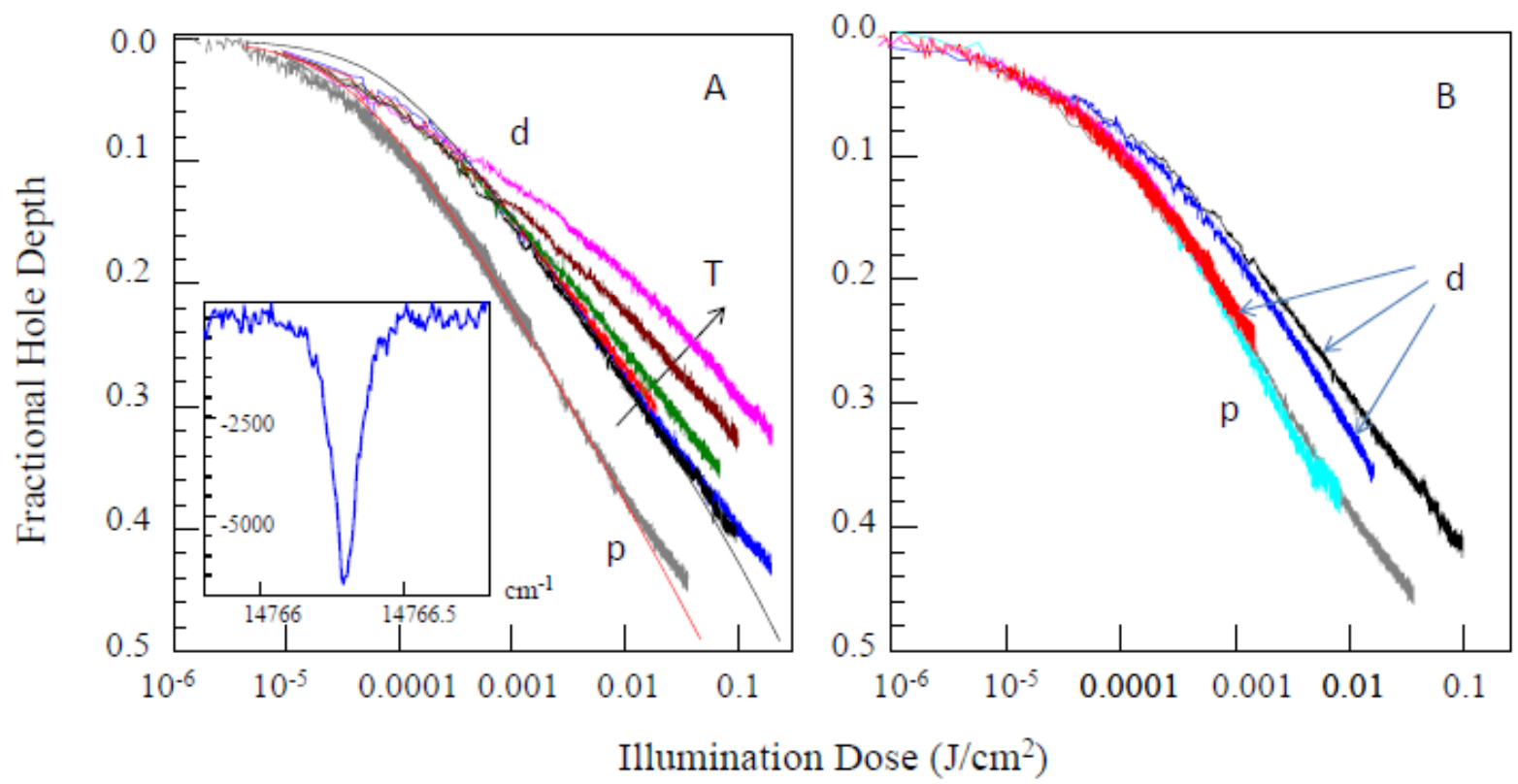
Figure 2. Frame A: Noisy curves: HGK curves obtained in OGP sample in deuterated environment at several temperatures from $5.5 \mathrm{~K}$ to $12.5 \mathrm{~K}$. Noisy grey curve is the $5.5 \mathrm{~K}$ curve for OGP sample in protonated environment and the same illumination intensity, $19 \mu \mathrm{W} / \mathrm{cm}^{2}$. Modeling results for $5.5 \mathrm{~K}$ curves are presented as well (smooth red curve: $\lambda=7.8 \ldots 12.0$, black curve: $\lambda=8.2 \ldots 13.1$ ). The insert in Frame A contains an example of a 20\%-deep hole measured in the sample featuring OGP detergent and deuterated solvent. Frame B: The 5.5 K HGK curves obtained for three different illumination intensities, $270 \mathrm{nW} / \mathrm{cm}^{2}, 2.3 \mu \mathrm{W} / \mathrm{cm}^{2}$ and $19 \mu \mathrm{W} / \mathrm{cm}^{2}$. Red, blue, black curves, respectively: deuterated Cyt b6f sample. Pink, light blue, grey curves: protonated sample. $0.001 \mathrm{~J} / \mathrm{cm}^{2}$ corresponds to about $50 \mathrm{sec}$ with the illumination intensity of 19 $\mu \mathrm{W} / \mathrm{cm}^{2}$. Black and grey experimental datasets are the same in both frames.

However, the observed difference in HGK is illumination intensity-dependent. Figure 2B shows several HGK curves obtained at $5.5 \mathrm{~K}$ for $\mathrm{Cyt}_{6} \mathrm{f}$ in protonated and deuterated solvent environment containing OGP detergent (same batch as in ${ }^{15}$ ). As can be seen, for protonated environment the burning rate does not depend on illumination intensity, within the intensity range employed in our experiments (pink, light blue and grey curves; the pink curve is almost invisible under the light blue, grey and red ones). Same burning rate is observed for the lowest burning intensity also in deuterated environment (red curve). In deuterated sample NPHB rate decreased with intensity. The highest intensity among those in Frame B $19 \mu \mathrm{W} / \mathrm{cm}^{2}$, was used to produce the curves in Frame A. Similar intensity effects were observed at other temperatures as well (not shown).

No major differences were observed in fixed-temperature recovery between protonated and deuterated Cyt $\mathrm{b}_{6} \mathrm{f}$ in OGP micelle, Figure $3 \mathrm{~A}$. The first 10-12 hours of recovery can be 
fitted using the same logic and similar parameters as in ${ }^{15}$. The recovery data is still in better agreement with $1 / \sqrt{V}$ barrier distribution shape than with Gaussian one. The latter would result in a faster initial recovery and slower recovery at the later stages. Significant amount of "spectral memory" (spectral holes refilling by burnt molecules returning to their pre-burn transition frequencies, not by "random" spectral diffusion) is present, as indicated by recovery rate depending on the fractional depth of the initial holes. This justifies the use of TLS approximation. The recovery appears to be only slightly slower for $\mathrm{Cyt}_{\mathrm{b}} \mathrm{b}_{6}$ in deuterated environment. Specifically, our data indicates an increase of the ground-state $\lambda_{g}$ by $0.8 \%$, clearly less than $8 \%$ obtained from the HGK curves when intensity effect shown in Figure 2B is ignored. This difference in recovery data is comparable to the range of day-to-day variations observed in different experiments for the protonated-solvent OGP samples, and likely reflects either the imperfections of our experiments or minor real differences due to the variations in sample's cooling history, for example. Thus, hole recovery results also indicate that intensity dependence and not the difference in $\lambda$ is responsible for observed differences in HGK for protonated and deuterated OGP-based samples. 


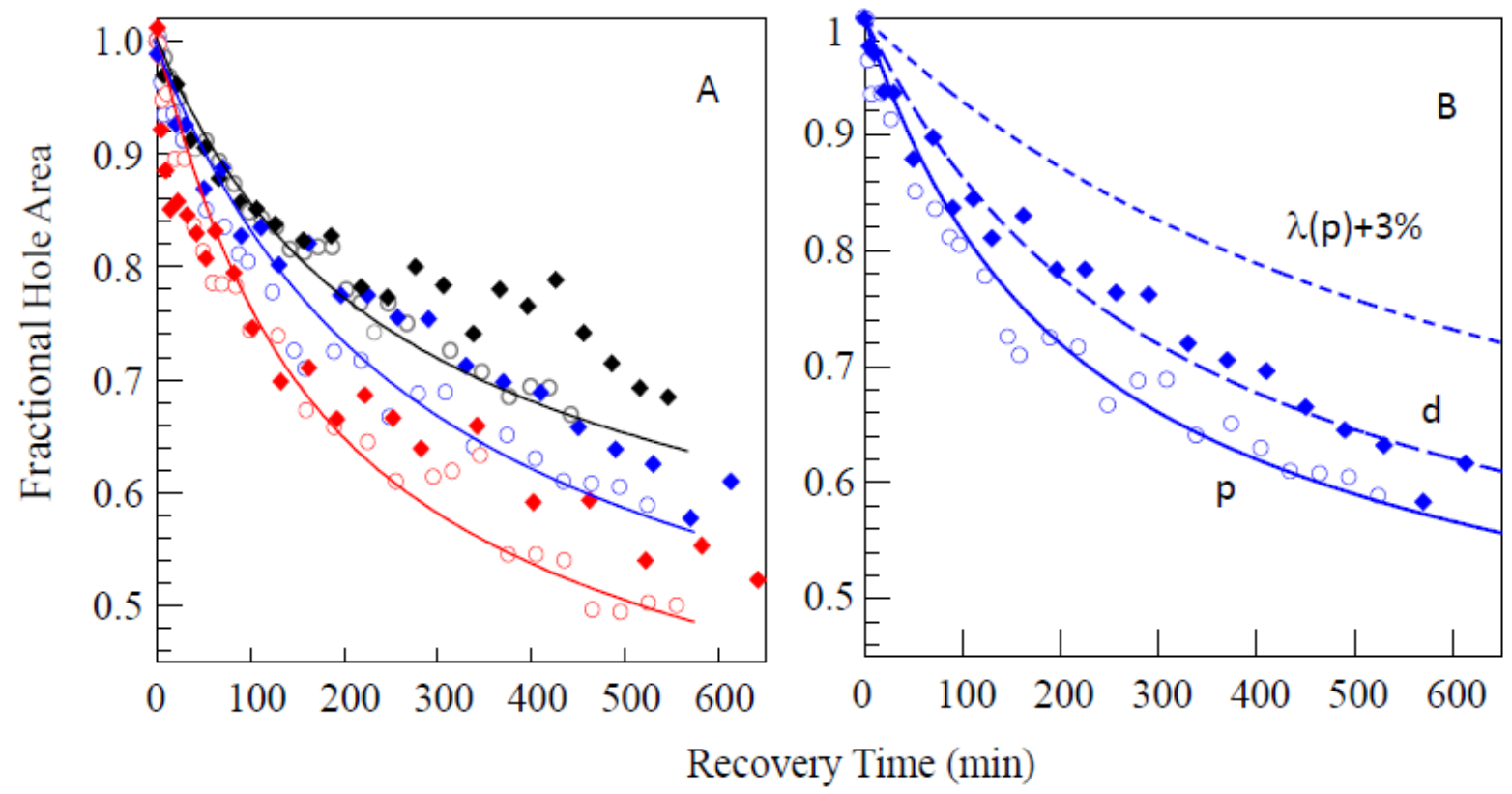

Figure 3: Frame A: Recovery at fixed burn temperature for $\mathrm{Cyt}_{6} \mathrm{~b}_{6} \mathrm{f}$ in regular/protonated (open circles) and deuterated (solid diamonds) environments. OGP sample. Red: 20\%-deep holes, blue: 30\% holes, Black: 40\% holes. Frame B: Symbols and solid blue line are the same as in Frame A. Long-dashed line represents the fit to the data for the deuterated OGP-based sample, corresponding to $0.8 \%$ increase of $\lambda$. Short-dashed blue line depicts recovery expected upon deuteration if the observed dynamics were due to $-\mathrm{OH}$ groups (increase of $\lambda$ upon deuteration by $3 \%)$.

Figure 4A shows thermocycling data for deuterated sample containing OGP detergent, compared to the data for the fixed-temperature recovery. Both spectral holes were originally 30\%-deep. The relative hole area is presented as a function of time rather than temperature. Some temperatures are shown next to the respective data points for clarity. The thermocycling dataset starts deviating from the fixed-temperature one at about $11 \mathrm{~K}$ and the recovery is complete by about $45 \mathrm{~K}$. Frame B depicts, as a function of temperature, the part of the barrier 
distribution that cannot be accounted for by the fixed-temperature recovery for both deuterated and protonated OGP samples. The black squares represent the result of division of the black square dataset from Frame A by the smooth blue curve representing the fit to the fixedtemperature recovery. Blue circles represent similar data for protonated sample. This type of data has to be fitted with $f=1-\int_{0}^{T_{\max }} g(T) d T$ to obtain the barrier distribution ${ }^{6,20}$. Here $g(T)$ is the distribution of the barrier heights on a temperature scale, $V(T)=k_{B} T \ln \left(t_{\max } \Omega_{0}\right)$ is barrier height on the energy scale, $t_{\max } \approx 60 \mathrm{sec}$ is time spent at the peak cycle temperature $T_{\max }$. With $7.6 \cdot 10^{12} \mathrm{GHz}$ attempt frequency one gets $\ln \left(\Omega_{o} t_{\max }\right)=33.75$. Similar data for protonated sample is also presented (blue circles). Overall, two datasets are fairly similar. As expected, deuteration is not supposed to influence the barrier heights $V$, but only the mass of the entity participating in conformational change, $m$. Note that possible errors of the hole area determination are large for high cycling temperatures when the holes are broadened and mostly recovered. Analysis of the two datasets suggests that recovery of both samples could be governed by a two-component barrier distribution (rather than one distribution with a stretched tail ${ }^{15}$ ). Examples of two $g(T)$ distribution components are shown with dashed lines in Figure 4B. The left side of the higherbarrier component is affected by the preceding recovery. These sample distributions, although reasonably realistic, are intended for qualitative illustrative purposes only and are not meant to be perfectly consistent with all available experimental data. Detailed discussion on the interplay between these two components, their shape and parameters, the possibility of one barrier distribution with negative correlation between $V$ and $m d^{2}$, degree of "spectral memory", the possibility of competitive NPHB, deficiencies of the WKB model, etc, will be presented elsewhere. 

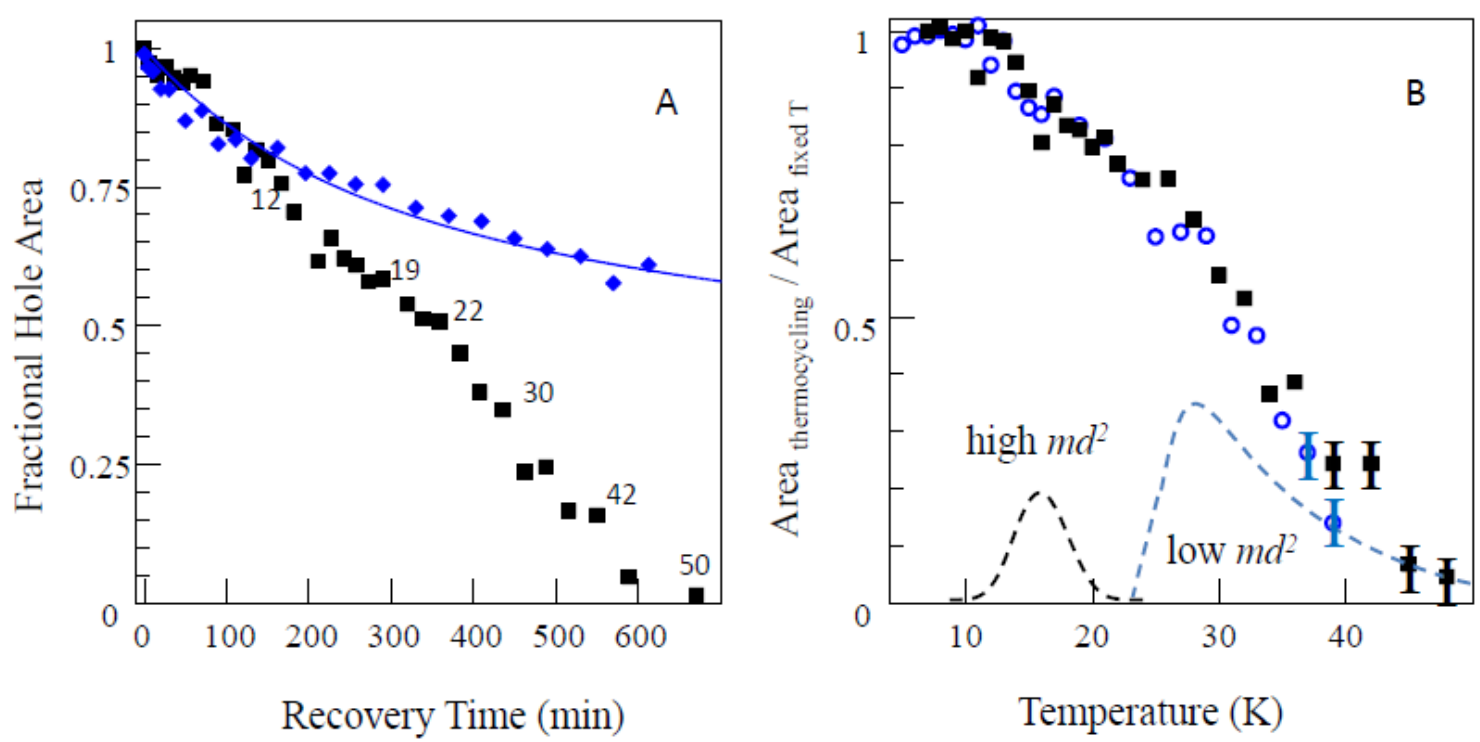

Figure 4: Frame A. Thermocycling-induced recovery for $\mathrm{Cyt}_{6} \mathrm{~b}_{6} \mathrm{f}$ in deuterated environment. OGP sample. Black squares: fractional hole area evolution upon thermocycling of a $30 \%$ hole. Cycling temperatures are indicated next to several datapoints. Blue diamonds: fixed-temperature recovery for a similar 30\%-deep hole. Blue curve: simulated fixed-temperature recovery, same as in Figure 3. Frame B: The (part of the) barrier distribution unaccounted for by the recovery at fixed burn temperature. Black squares: deuterated OGP sample. Open blue circles: protonated OGP sample. Dashed lines represent two components of the barrier distribution and are intended as a qualitative illustration only.

3.2. Detergent Effects: Qualitatively similar results were observed also for the samples from the new Cyt $b_{6} f$ sample batch produced with DM detergent. Absorption and fluorescence spectra of OGP and DM samples are compared in Figure 5A. Absorption spectra have nearly identical shape and parameters. $\triangle \mathrm{FLN}$ spectra of the DM sample are shown in Figure $5 \mathrm{~B} . \triangle \mathrm{FLN}$ spectrum of the OGP sample obtained with $680 \mathrm{~nm}$ excitation is shown for comparison. Strong red shift of the non-resonantly excited fluorescence, Figure 6A, and the difference in the shape of the $\Delta \mathrm{FLN}$ 
spectra, Figure 6B, indicate significant increase of electron-phonon coupling in DM samples. The $\Delta$ FLN spectrum for DM sample and $680 \mathrm{~nm}$ excitation (black) exhibits broader phonon sideband than the corresponding spectrum of the OGP sample (green), which is to be expected for higher electron-phonon coupling when multi-phonon processes are taken into account. (Also, $\Delta$ FLN spectra for 670 and $680 \mathrm{~nm}$ excitation are significantly different, which indicates the presence of energy transfer between the two chlorophylls of the dimer.) We were unable to burn more than 32\% zero-phonon holes in the spectra of the DM-based sample, which indicates that Huang-Rhys factor $S$ for phonons is about 1.1. According to modeling, increase of $S$ from 0.72 (in OGP samples ${ }^{15}$ ) to 1.12 must result in about four-fold slowdown of spectral hole burning, if all other model parameters were staying the same.
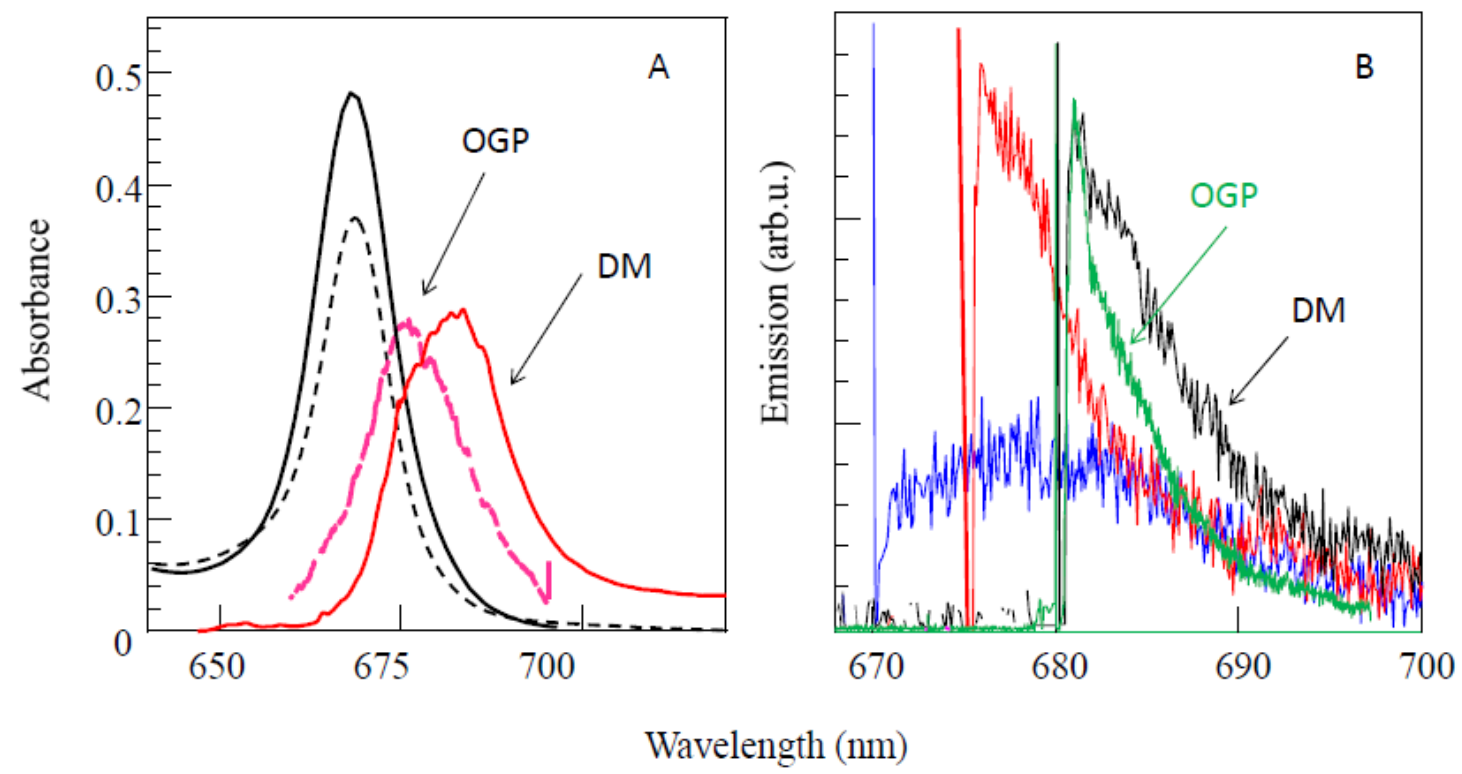

Figure 5. Frame A: Absorption (black) and emission (red/pink) spectra of OGP (dashed line) and DM (solid line) based samples at $5 \mathrm{~K}$. Frame B: $\Delta$ FLN spectra for DM-based sample obtained with illumination at $670 \mathrm{~nm}$ (blue), $675 \mathrm{~nm}$ (red) and $680 \mathrm{~nm}$ (black). Green curve is the $\Delta \mathrm{FLN}$ spectrum of the OGP-based sample, $680 \mathrm{~nm}$ excitation. 

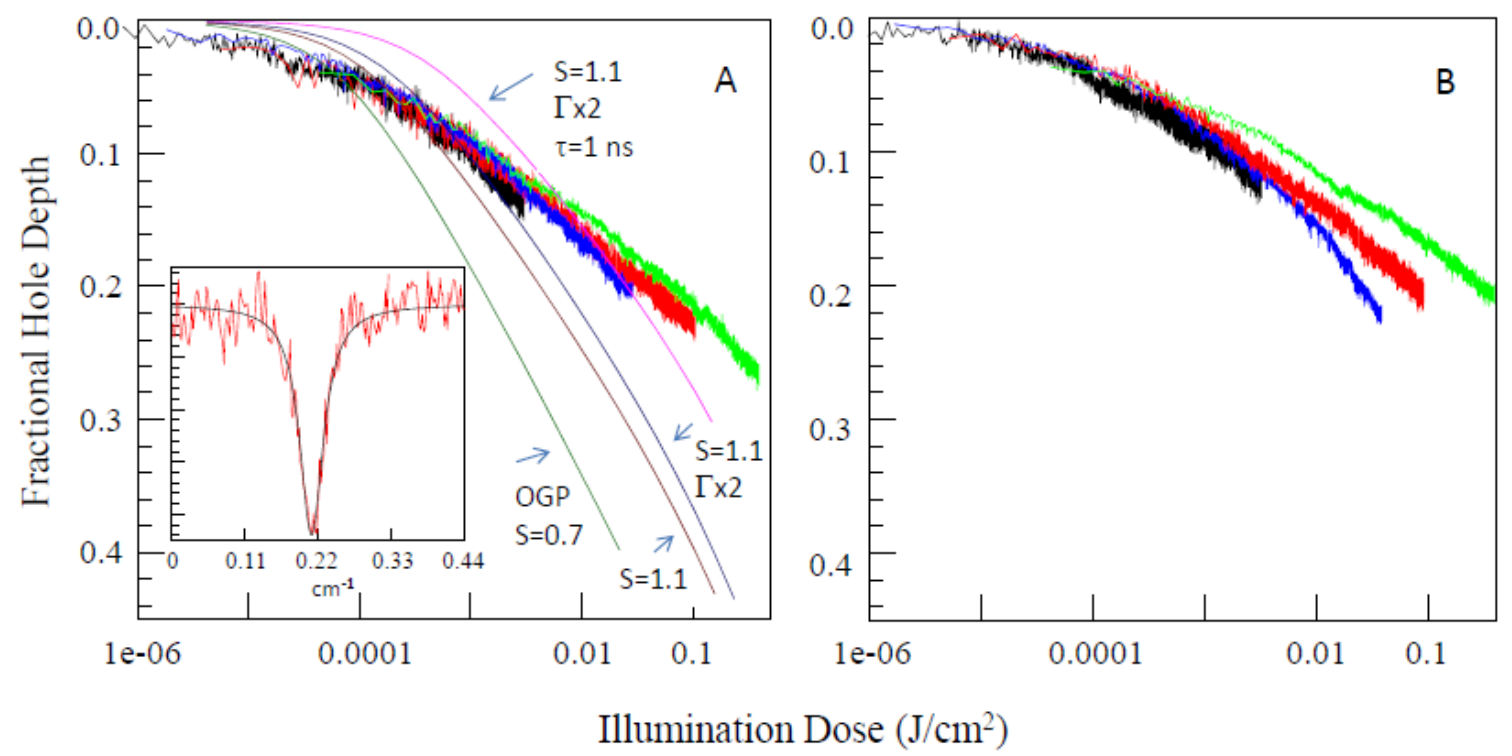

Figure 6. Frame A. 5 K HGK curves for different illumination intensities for protonated solvent DM-based sample. Frame B: Same for deuterated DM-based sample. The excitation intensities were $400 \mathrm{nW} / \mathrm{cm}^{2}, 3.5 \mu \mathrm{W} / \mathrm{cm}^{2}, 28 \mu \mathrm{W} / \mathrm{cm}^{2}$ and $214 \mu \mathrm{W} / \mathrm{cm}^{2}$ for black, blue, red and green curves, respectively. The insert in Frame A contains an example of a shallow hole at $2 \mathrm{~K}$.

However, NPHB appears to be more than ten times slower in both protonated and deuterated versions of the DM-containing sample, Figure 6. (Note also the more pronounced intensity dependence of the HGK for deuterated sample, Frame B. Thus, this effect is present regardless of the detergent micelle composition). Additional slowdown of burning could be explained by DM-based sample containing a smaller fraction (or none) ${ }^{31}$ of Cyt $\mathrm{b}_{6} \mathrm{f}$ with $\mathrm{Chl} a$ lifetimes not reduced by quenching by aromatic groups. Note that DM was used as a detergent in Cyt $b_{6} f$ samples where quenching was previously reported ${ }^{34-36}$. Reduction of the lifetime from several ns to $\sim 200$ ps ${ }^{34-36}$ would easily explain ten-fold slowdown of burning, even without an increase of electron-phonon coupling (see Eq. 2). The homogeneous line widths in DM sample were determined at several temperatures from 2 to $10 \mathrm{~K}$ and they were systematically $\sim 0.01 \mathrm{~cm}^{-1}$ 
larger than those observed in OGP sample ${ }^{15}$. On the other hand, results obtained at $2 \mathrm{~K}$ are still suggesting lifetimes longer than 200 ps from ${ }^{34-36}$. Lifetime of 200 ps corresponds to homogeneous line width of $0.0265 \mathrm{~cm}^{-1}$. The narrowest linewidths observed for DM sample at 2 $\mathrm{K}$ are about $0.0165 \mathrm{~cm}^{-1}$. A $10 \%$-deep $0.04 \mathrm{~cm}^{-1}$ wide hole $\left(0.02 \mathrm{~cm}^{-1}\right.$ line width $)$ is shown in the insert in Figure 6A. The $2 \mathrm{~K}$ line/hole widths still contain some contribution from pure dephasing, therefore 320 ps is just the shorter limit of the dephasing-free lifetime in DM samples. Attributing the $\sim 0.01 \mathrm{~cm}^{-1}$ difference between homogeneous line widths in OGP and DM samples to quenching, one can obtain the average quenching time of about 530 ps. Smooth dark green curve in Figure 6A represents a fit to the OGP sample data ${ }^{15}$. Smooth brown curve results from an increase of Huang-Rhys factor $S$ from 0.72 to 1.12, all other model parameters staying the same. Smooth blue curve results from an additional two-fold increase of the homogeneous line width if this increase is attributed strictly to pure dephasing. Apparently, this is not enough. Some decrease of the dephasing-free lifetime (e. g. due to quenching) is required to explain the slowdown of burning without increasing the mean of the $\lambda$-distribution (smooth magenta curve corresponds to the decrease of $\tau_{\mathrm{fl}}$ from $2.5 \mathrm{~ns}$ to $1 \mathrm{~ns}$.). Experimental results in Figure 6 also suggest significantly broader distribution of NPHB rates than was observed for OGP-based sample and implied in above modeling. This increase could be attributed to either genuinely broader $\lambda$-distribution (but see next paragraph) or to the distribution of the excited state lifetimes (in this case - quenching times). As demonstrated in ${ }^{37}$, introduction of the linewidth distribution indeed is expected to broaden the distribution of the NPHB yields.

The fixed-temperature hole recovery in DM-based sample is compared to the recovery in OGP-based sample in Figure 7A. The recovery of $20 \%$ holes proceeds at nearly identical rate in both samples. The recovery of a 30\% hole was significantly slower in DM-based sample than in 
OGP-based sample. However, one should take into account that 30\%-deep holes burnt into the spectra of DM sample (S 1.12) are much closer to saturation than 30\%-deep and even 40\%-deep holes for OGP-based sample ( $S \sim 0.72$ ) and consequently partial $\lambda$ - (or barrier) distributions encoded into the holes have larger contributions from larger $\lambda$. (Assuming that one starts from the same true full barrier distributions prior to burning in all cases.) Thus, slowdown of the fixedtemperature recovery for nearly-saturated holes in DM-based samples is ultimately attributable to the increase of $S$ and does not require any change in the full true barrier distribution before burning. The recovery of 30\%-deep holes upon thermocycling was similar in protonated and deuterated DM-based samples. Figure 7B shows hole area as a function of cycling temperature, corrected for fixed-temperature recovery that would occur during the experiment. Thermocycling behavior of the DM-based samples was also similar to that of OGP-based samples (Figure 4) except that in the case of the DM-based sample some fraction of the hole persists till slightly higher temperatures. This is again expected if one remembers that 30\% holes in DM-based sample are much more saturated than $30 \%$ holes in OGP-based sample and contain larger relative contribution from larger $\lambda$ and $V$. The full distribution of the barrier heights is unlikely affected by the choice of the detergent; these are the sub-distributions encoded into the holes of equal fractional depth that are different between OGP- and DM-based samples. Both protonated and deuterated DM-based samples feature a fast recovery step at around 11-15 K, just like OGPbased samples, indicating that two-component barrier distributions encoded into the holes is a general feature of Cyt ${ }_{6} 6$, independent on detergent. The insert in Figure 7B contains examples of the hole spectra - right after burning (blue), after one hour of recovery at $5 \mathrm{~K}$ (red) and after cycling to $41 \mathrm{~K}$ (black). 

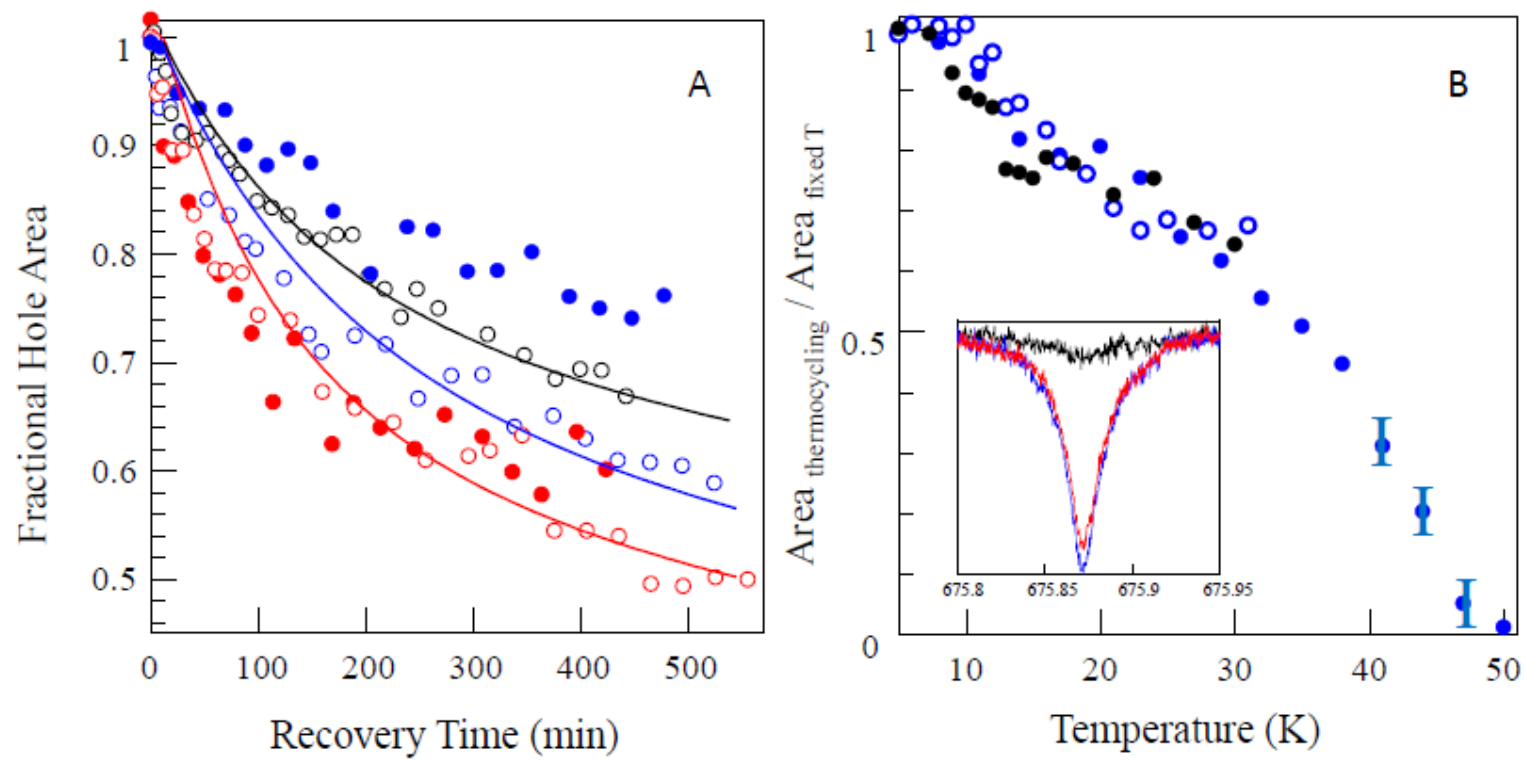

Figure 7: Frame A: Fixed-temperature recovery of 20\%-deep (red circles) and 30\%-deep holes (blue circles) in DM-based sample, protonated solvent. Open circles show results for 20\% (red), $30 \%$ (blue) and 40\% (black) holes for OGP-based sample and respective simulated curves (solid lines). Red: $20 \%$ hole. Blue: $30 \%$ hole. Black: $40 \%$ hole. Frame B: Hole area as a function of maximal cycle temperature in several DM-based samples, including deuterated one (black), 30\% hole, protonated solvent. The insert shows examples of the hole spectra: immediately after burning (blue); after one hour at $5 \mathrm{~K}$ (red) and after cycling to $41 \mathrm{~K}$ (black).

3.3. Chlorophyll in water/ glycerol glass. In order to explore the possibility of protein dynamics "slaving" to that of the surrounding glass, we attempted to directly examine the spectral dynamics of buffer-glycerol mixture employed as a solvent and glass-forming medium in experiments on protein (see Figure 1B). Absorption and emission spectra of Chl $a$ in protonated and deuterated water / glycerol / methanol $(>30 \% />60 \% /<10 \%)$ mixtures at $5 \mathrm{~K}$ are depicted in Figure 8. Absorption spectra exhibit the main $\mathrm{Q}_{\mathrm{y}}$ band at about $670 \mathrm{~nm}$ and a noticeable shoulder at $715 \mathrm{~nm}$. The relative intensity of the $715 \mathrm{~nm}$ shoulder was gradually 
increasing at the expense of the $670 \mathrm{~nm}$ band when the sample was left at room temperature for extended periods of time. This redistribution process could be partially reversed by sonication. Also, the shoulder was relatively more intense for samples with higher $\mathrm{Chl} a$ concentration. Thus, we attribute this shoulder to aggregated $\mathrm{Chl} a$. Observed manifestations of the aggregates are similar to those reported for $10 \%$ acetonitrile or DMF to $90 \%$-water mixtures in ${ }^{38}$. Absorption bands at $\sim 740 \mathrm{~nm}{ }^{39}$ were never observed in our experiments.
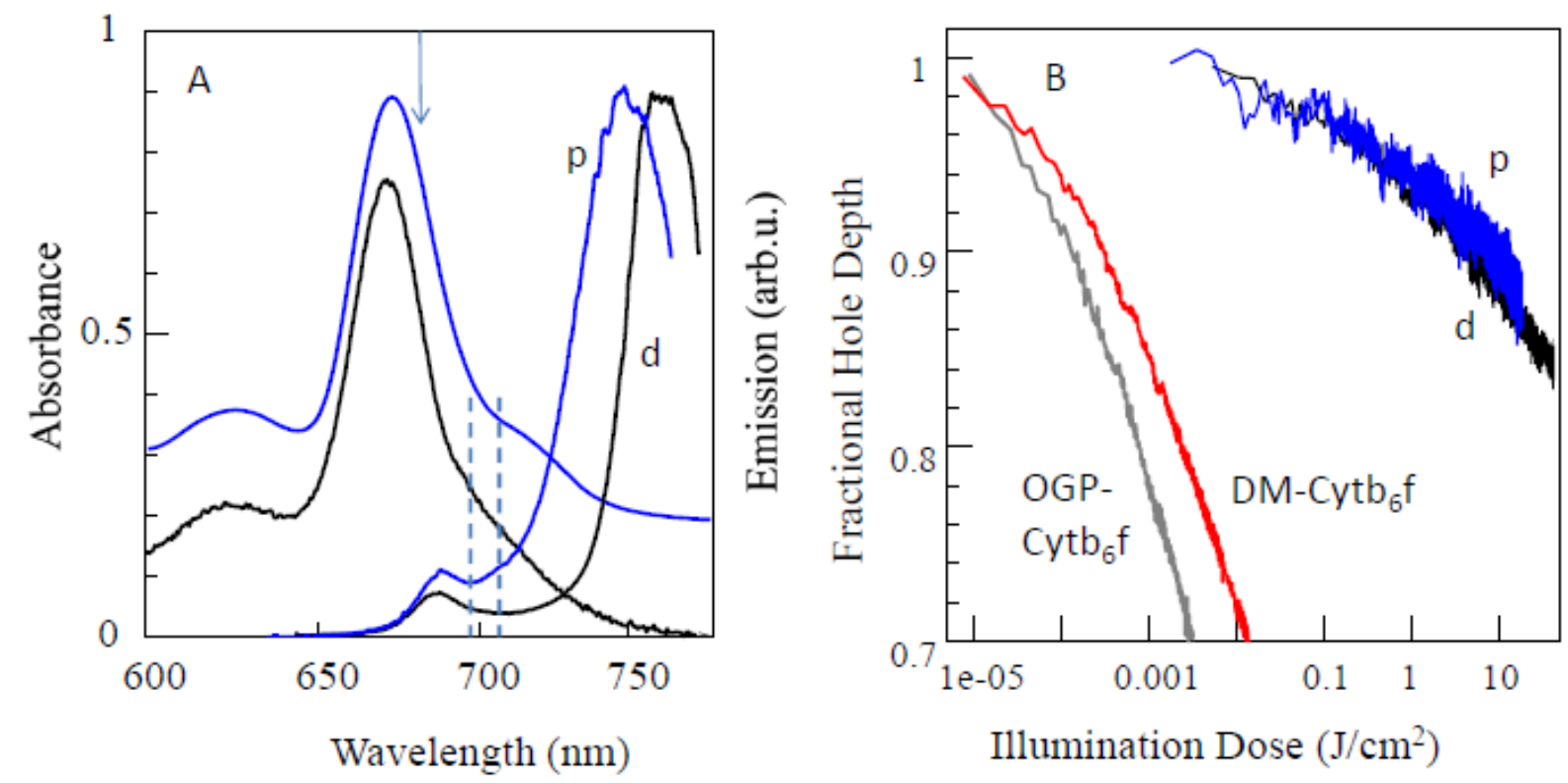

Figure 8: Frame A: Representative absorption and emission spectra of Chl $a$ in water / glycerol mixture with $<10 \%$ methanol. Black: deuterated solvent. Blue: protonated solvent. Absorption spectrum of protonated sample is shifted up for clarity. Dashed vertical lines indicate the detection window for NPHB experiments in fluorescence excitation mode. Arrow indicates the hole-burning wavelength. Frame B: HGK curves for protonated (blue) and deuterated (black) solvent after correction for contributions from long-wavelength emission band within the detection window. Also shown, for comparison, are 5 K HGK curves for OGP (grey) and DMbased (red) Cyt b6f samples. 
Aggregation is causing the red shift of the absorption due to excitonic coupling, as in the case of the "red antenna states" of Photosystem I, for example ${ }^{40}$. The emission spectra exhibit strong aggregate fluorescence at $\sim 750-760 \mathrm{~nm}$ and weak monomer fluorescence at $\sim 685 \mathrm{~nm}$. Large shift of the aggregate fluorescence with respect to the absorbance indicates strong electron-phonon coupling and suggests that aggregates would not contribute much to the resonant NPHB. The emission spectra for supposedly similar samples produced on different days exhibited some variability in terms of peak positions and intensity ratios. Aggregate fluorescence was much stronger than monomer fluorescence in all samples, despite $\sim 670 \mathrm{~nm}$ absorption band featuring several times higher OD than the aggregate shoulder. Thus, there is strong quenching of the chlorophyll monomer fluorescence by water-glycerol mixture at cryogenic temperatures. This is consistent with room temperature results for $\mathrm{Chl} a$ in the mixtures of organic solvents and water $^{39}$. To probe monomeric $\mathrm{Chl} a$ selectively in fluorescence excitation mode, fluorescence was detected using 695-710 nm bandpass filter. By trapping Chl $a$ in PVA gels and then allowing the gels to absorb water and glycerol we could also achieve situation where monomer Chl $a$ fluorescence was quenched but no aggregate fluorescence at around $750 \mathrm{~nm}$ was observed. Thus, aggregation and quenching of the $685 \mathrm{~nm}$ fluorescence in water-glycerol Chl $a$ solution are independent phenomena. NPHB in the Chl $a$ monomer band appeared to be very inefficient in both protonated and deuterated samples. About $10 \%$ holes could be burnt in a couple of hours using light intensities of about $4 \mathrm{~mW} / \mathrm{cm}^{2}, 200$ times higher than the highest intensities reported for Cyt $b_{6} f$ in Figure 2 and 20 times higher than in Figure 6. In all experiments deuterated samples exhibited even somewhat faster burning than protonated ones. This, however, was most likely due to different contributions of aggregate fluorescence to total fluorescence within the 
detection window. As the aggregate fluorescence peak in protonated sample is at somewhat shorter wavelength, one can suggest that about half of the fluorescence in the $695-710 \mathrm{~nm}$ detection window belongs to the monomers. Frame B of Figure 8 shows HGK curves for Chl $a$ in protonated and deuterated solvent, as well as $5 \mathrm{~K} \mathrm{HGK}$ curves for $\mathrm{Cyt}_{6} \mathrm{f}$ for comparison. Thus, deuteration does not slow down the NPHB process in chlorophyll in water / glycerol mixtures. Due to poor fluorescence and NPHB yields and signal to noise ratio, we have not attempted fixed temperature recovery or thermocycling experiments on Chl $a$ in solution so far (work in progress).

\section{Discussion.}

4.1 Nature of the tunneling entities. Fitting various recovery results using one single barrier or $\lambda$-distribution as suggested in ${ }^{15}$ ultimately proved impossible (the details may be presented elsewhere). Thus, spectral hole burning in Cyt $\mathrm{b}_{6} \mathrm{f}$ may involve two tiers of the protein energy landscape featuring degenerate distributions of the NPHB yields (or excited-state $\lambda$ ), with similar yields resulting from different combinations of $m d^{2}$ and $V$ (see for example Figure 4B). (At the moment we cannot exclude an alternative scenario involving one energy landscape tier with negatively correlated barrier and $m d^{2}$ distributions. While simple TLS model in Figure 1 provides no reasons for such a correlation, the presence of a distribution of $m d^{2}$ values for the same tier of the energy landscape would point to cooperative effects. The numerical values for $m d^{2}$ reported below would then represent the limits of the range of possible $m d^{2}$ values.) For thermocycling the lower-barrier higher $m d^{2}$ part is the first to recover. This component features $m d^{2}$ of about $4 \cdot 10^{-46} \mathrm{~kg} \cdot \mathrm{m}^{2}$ as can be determined from the modeling of the fast recovery step observed in thermocycling experiments at 11-15 K (Figure 4). This value is too large to be 
ascribed to the tunneling of a single proton in a double-well potential associated with a hydrogen bond in either the protein or glassy solid surrounding protein. We note that a "photoconversion" hole burning mechanism involving rearrangement of a hydrogen bond between protein and chlorophyll was proposed in the core antenna complexes of Photosystem II ${ }^{41-43}$. Cooperative effects involving the large hydrogen bonds network of the protein are still possible, since in the case of the cooperative effects $m$ becomes uncoupled from the mass of some one particular atom or chemical group. For a single methyl or hydroxyl group $m d^{2} \sim 4 \cdot 10^{-46} \mathrm{~kg} \cdot \mathrm{m}^{2}$ would mean a displacement $d$ of $\sim 1 \AA$. The $m d^{2}$ value for the second component, dominating fixed-temperature recovery, could be inferred from thermocycling data for higher temperatures and the data on the very beginning of the NPHB process in Figure 2 that suggests $m d^{2} \approx 1.5 \cdot 10^{-46} \mathrm{~kg} \cdot \mathrm{m}^{2}$ for systems involved in the initial hole burning and featuring the lowest $\lambda_{\text {exc }}$.

HGK and recovery results obtained for $\mathrm{Cyt}_{6} \mathrm{f}$ in deuterated buffer / glycerol environment indicate almost no deuteration effects. This clearly rules out the possibility that any spectral dynamics is due to proton / deuteron tunneling in the outside environment, including the scenarios involving cooperative effects. Since $\lambda \sim \sqrt{m}$, increasing $m$ by a factor of two would result in an increase of $\lambda$ by a factor of $\sqrt{2}$. Depending on the range of $\lambda$ (different combinations of matching $\lambda$ and attempt frequency $\Omega_{0}$ would produce the same HGK curves, see Eq. 2), the NPHB yield and fixed-temperature recovery rates would be reduced 50-1000 times. The methyl groups change their mass upon full deuteration from 15 to $18 \mathrm{~g} / \mathrm{mole}$ and changes in their tunneling dynamics would be easily noticeable (9.5\% change in $\lambda$ and $~ 50$ times change in fixed-temperature recovery rate for $\left.\lambda_{g} \approx 20\right)$. The mass of the $-\mathrm{OH}$ group changes by $6 \%$, resulting in a $3 \%$ change of $\lambda$. Results obtained for chlorophyll in water / glycerol environment exclude the possibility of proton / deuteron tunneling affecting spectral dynamics but, taking into 
account poor quality of this data, could still be consistent with the tunneling involving $-\mathrm{OH}$ groups. Could any dynamics observed for $\mathrm{Cyt}_{\mathrm{b}} \mathrm{f}$ still originate from the $-\mathrm{OH}$ group tunneling in the outside solution? The Cyt $b_{6} f$ data is of significantly higher quality than chlorophyll in solution data. It is unlikely that we could miss a $3 \%$ change in $\lambda$ in Cyt $b_{6} f$. For instance, if $\lambda$ were increased by $3 \%$, the recovery of a $30 \%$ hole in the deuterated OGP-detergent sample in Figure 3 would become slower than what was observed for a $40 \%$ hole (short-dashed blue curve in Figure 3B). As fixed-temperature recovery is preferentially probing the smaller- $m d^{2}$ tier of the barrier distribution, one can be confident that this tier belongs to the protein and likely reflects cooperative dynamics of the hydrogen bond network. Note that fixed-temperature $(5 \mathrm{~K})$ recovery does not directly probe the second, higher- $m d^{2}$ lower- $V$ component in Figure 4B, at least within the first 30 hours. Thus, one should be somewhat cautious about claiming its deuterationindependence. However, any deuteration-related effect, if present for this component, cannot possibly be strong, or this component would shift towards higher $\lambda$ and would not contribute to NPHB and recovery of the deuterated samples. This would in turn result in a decrease of the apparent NPHB rate for all illumination intensities (second burning channel rendered much slower in competitive burning model, the only channel rendered much slower for some molecules in one-tier-different- $m d^{2}$ model) and in significant differences in thermocycling (smaller, lower T contribution missing / reduced). These effects were not observed. Given the location of the chlorophylls within the Cyt $\mathrm{b}_{6} \mathrm{f}$ (see Figure 1B) and the presence of the detergent micelle surrounding the hydrophobic part of the protein in the vicinity of the chlorophylls, chlorophylls inside the protein reporting the dynamics of the $-\mathrm{OH}$ groups of the outside environment is not very likely. Presence of a strong detergent effect also suggests that observed dynamics is that of the protein or perhaps protein-in-detergent-micelle rather than that of the 
outside solvent. Strong quenching of Chl $a$ monomer fluorescence in solution and the absence of such strong quenching in protein (the quenching possibly observed in DM-based samples is still two orders of magnitude weaker than quenching in water / glycerol mixture, see Figure $8 \mathrm{~B}$ ) is still another strong argument against chlorophyll in Cyt $b_{6} \mathrm{f}$ being somehow exposed to water / glycerol mixture (and therefore exhibiting "glassy" dynamics). The $0.8 \%$ difference in $\lambda$ between protonated and deuterated samples may be a measure of the imperfections of our experiment or reflect real minor differences in sample preparation and cooling history. As shown in ${ }^{44}$, cooling history may affect how far the pigment-protein systems are from thermal equilibrium and consequently introduce minor variations in the apparent rates of NPHB and of recovery.

\subsection{Intensity Effect in HGK of the Deuterated Cyt bof Samples. The apparent NPHB}

rate may be affected by the changes in the balance between hole recovery occurring during burning (intensity-independent process) and burning process per se. However, in this scenario one would expect faster burning (as a function of $\mathrm{J} / \mathrm{cm}^{2}$, as depicted in all our HGK figures) for higher intensity. The same photon numbers would be delivered in a shorter time, which should tilt the balance in favor of burning. Even for $200 \mu \mathrm{W} / \mathrm{cm}^{2}$ intensity (Figure 6), we are several orders of magnitude away from the saturating intensities. It was noticed for both $\mathrm{CP} 43^{14}$ and Cyt $\mathrm{b}_{6} \mathrm{f}$ in protonated environment ${ }^{15}$ that (all other parameters, such as intensity, being the same) hole burning slows down with the increase of temperature, in agreement with the homogeneous line width increasing with temperature and the peak absorption cross-section decreasing accordingly. Similar behavior was observed also for Cyt $\mathrm{b}_{6} \mathrm{f}$ in the deuterated environment (Figure 2A). Thus, slowdown of burning with increasing illumination intensity may be explained by local heating of the immediate protein environment of the chlorophyll molecules by 
several Kelvin with respect to the "global" temperature of the sample, measured by the macroscopic sensor. For the proposed deuteration-dependent local heating effect to be observable, the bulk thermal conductivity and/or the protein / solvent interface thermal conductance have to be significantly lowered in the case of deuterated solvent. Deuterated substances generally have somewhat lower heat conductivities than their protonated counterparts, thus making some kind of increased local heating more plausible. Comparison of cryogenic-temperature data for protonated and deuterated versions of the same compound appears to be available only for ethanol ${ }^{45}$, and depending on the phase the values are in the range between 0.1 and $1 \mathrm{~W} / \mathrm{m} \cdot \mathrm{K}$. The low-temperature heat conductivity of the deuterated ethanol is indeed somewhat lower than of the regular ethanol. Heat conductivities of either amorphous ice or glycerol at cryogenic temperatures are also in the range of $0.1-1 \mathrm{~W} / \mathrm{m} \cdot \mathrm{K}^{46,47}$. The data on various water / cryoprotectant mixtures as well as various tissues is available in ${ }^{48}$ but only down to about $120 \mathrm{~K}$. However, in this range the heat conductivities of the mixtures lay between those of ice and pure glycerol. Similar heat conductivity values were calculated for GFP and myoglobin in ${ }^{49}$. Careful analysis of a number of steady-state and dynamic scenarios (see Supplemental information section) indicates, however, that explaining our results requires several order of magnitude lower bulk heat conductivity values. The remaining possibilities involve higher thermal resistance of the interface between (non-modified, protonated) protein and deuterated outside environment, for instance due to acoustic mismatch. The calculated values of the interface thermal conductance between protein and water are in the $100 \mathrm{MW} /\left(\mathrm{K}^{2}{ }^{2}\right)$ range at room temperature ${ }^{50}$. The cryogenic-temperature surface heat conductance data is scarce and usually pertains to two solid objects compressed or soldered together. For example, the $90 \mathrm{~K}$ $0.2 \mathrm{MPa}$ interface thermal conductance between copper and aluminum nitride is about $300 \mathrm{~W} / \mathrm{K}$ 
$\mathrm{m}^{2}$, with a strong decreasing trend towards lower temperatures ${ }^{51}$. The values necessary to explain our results are on the order of $10 \mathrm{~W} / \mathrm{Km}^{2}$. One should note, however, that for objects that are as small as individual $\mathrm{Cyt}_{\mathrm{b}} \mathrm{f}$ dimer, continuum approach to the space inside and outside the protein / amorphous solid interface is not sufficient, and one has to look into exact molecular nature of the relevant vibrations. Vibrations of proteins are quite distinct from those of threedimensional amorphous materials. Most vibrational modes of harmonic aqueous glass are extended over large volume. ${ }^{52,53}$ Most vibrational modes of a harmonic protein, however, are localized to a relatively small region of the protein. ${ }^{54-56}$

\section{Conclusions.}

Spectral dynamics of $\mathrm{Cyt}_{6} \mathrm{~b}_{6} \mathrm{f}$ manifesting in NPHB experiments appears to be nearly independent of the deuteration of the buffer / glycerol matrix containing the protein (apart from some differences in heat dissipation), and of the detergent employed in the isolation and purification procedure. Apparent differences in NPHB and hole recovery rates between samples with different detergent are attributable to DM-based dimeric Cyt $b_{6} f$ samples featuring stronger electron-phonon coupling and shorter (and possibly distributed) excited state lifetime, in agreement with results obtained for DM-based Cyt $b_{6} f$ samples from other organisms by other groups. Two plausible explanations for spectral dynamics remain. The observed dynamics most likely is the genuine protein dynamics, despite the shape of the barrier distribution(s) resembling $\sim 1 / \sqrt{V}$, and likely involves cooperative effects. Alternatively, but less likely one of the two subdistributions of barriers observed in $\mathrm{Cyt}_{6} \mathrm{~b}_{6} \mathrm{f}$ may be due to the outside solvent environment dynamics involving specifically the whole $-\mathrm{OH}$ groups. Single proton / deuteron tunneling in the water / glycerol environment can be safely excluded as the origin of our NPHB observations. 
The differences in heat dissipation between protonated and deuterated samples are most consistent with the presence of higher interface thermal resistivity between (still protonated) protein and deuterated outside environment, possibly due to phonon frequency mismatch.

\section{Acknowledgements}

Work at Concordia was supported by NSERC, CFI and Concordia University. R.P. and M.A.L. acknowledge support by the Ministry of Economy and Competiveness of Spain (Grant No. AGL2014-55300-R) and Aragon Government (Grant E33).

\section{Supporting Information Available:}

Various estimates for heat conductivity required to explain local heating of the protein by laser illumination. This material is available free of charge via the Internet at http://pubs.acs.org. 


\section{References.}

1. Wagie, H. E.; Geissinger, P. Hole-Burning Spectroscopy as a Probe of Nano-environments and Processes in Biomolecules: A Review, Appl. Spectrosc. 2012, 66, 609-627.

2. Berlin, Y.; Burin, A.; Friedrich J.; Köhler, J. Spectroscopy of Proteins at Low Temperature. Part I: Experiments with Molecular Ensembles, Physics of Life Reviews 2006, 3, 262-292.

3. Jankowiak, R.; Hayes, J. M.; Small, G. J. Spectral Hole-Burning Spectroscopy in Amorphous Molecular Solids and Proteins, Chem. Rev. 1993, 93, 1471-1502.

4. Köhler, W.; Friedrich, J. Distribution of Barrier Heights in Amorphous Organic Materials, Phys. Rev. Lett. 1987, 59, 2199-2202.

5. Köhler, W.; Meiler, J.; Friedrich, J. Tunneling Dynamics of Doped Organic Lowtemperature Glasses as Probed by a Photophysical Hole-burning System, Phys. Rev. B 1987, $35,4031-4037$.

6. Köhler, W., Friedrich, W., Scheer, H., Conformational Barriers in Low-temperature Proteins and Glasses. Phys. Rev. A 1988, 37, 660-662.

7. den Hartog, F. T. H.; van Papendrecht, C.; Störkel, U.; Völker, S. Protein Dynamics in Photosystem II Complexes of Green Plants Studied by Time-Resolved Hole-Burning, J. Phys. Chem. B 1999, 103, 1375-1380.

8. Anderson, P.W.; Halperin, B. I.; Varma, C. M. Anomalous Low-temperature Thermal Properties of Glasses and Spin Glasses, Phil. Mag. 1972, 25, 1-9.

9. Phillips, W.A. Tunneling States in Amorphous Solids, J. Low. Temp. Phys. 1972, 7, 351-360.

10. Frauenfelder, H.; Sligar, S. G.; Peter, G. The Energy Landscapes and Motions of Proteins, Science 1991, 254, 1598-1603. 
11. Fenimore, P. W.; Frauenfelder, H.; McMahon, B. H.; Young, R. D. Proteins are Paradigms of Stochastic Complexity, Physica A 2005, 351, 1-13.

12. Hofmann, C.; Aartsma, T. J.; Michel H.; Köhler, J. Direct Observation of Tiers in the Energy Landscape of a Chromoprotein: A Single-molecule Study, Proc. Natl. Acad. Sci. USA 2003, 100, 15534-15538.

13. Berlin, Y.; Burin, A.; Friedrich, J.; Köhler, J. Low Temperature Spectroscopy of Proteins. Part II: Experiments with Single Protein Complexes, Phys. Life Rev. 2007, 4, 64-89.

14. Najafi, M.; Herascu, N.; Seibert, M.; Picorel, R.; Jankowiak, R.; Zazubovich, V. Spectral Hole Burning, Recovery, and Thermocycling in Chlorophyll-Protein Complexes: Distributions of Barriers on the Protein Energy Landscape, J. Phys. Chem. B 2012, 116, 11780-11790.

15. Najafi, M.; Herascu, N.; Shafiei, G.; Picorel, R.; Zazubovich, V. Conformational Changes in Pigment-Protein Complexes at Low Temperatures - Spectral Memory and a Possibility of Cooperative Effects, J. Phys. Chem. B 2015, 119, 6930-6940.

16. Reinot, T.; Dang, N. C.; Small, G. J. A Model for Persistent Hole Burned Spectra and Hole Growth Kinetics that Includes Photoproduct Absorption: Application to Free Base Phthalocyanine in Hyperquenched Glassy Ortho-dichlorobenzene at $5 \mathrm{~K}$, J. Chem. Phys. 2003, 119, 10404-10414.

17. Reinot, T.; Small, G. J. Modeling of Dispersive Nonphotochemical Hole Growth Kinetics Data. Al-phthalocyanine Tetrasulphonate in Hyperquenched Glassy Water, J. Chem. Phys. 2000, 113, 10207-10214. 
18. Reinot, T.; Hayes, J. M.; Small, G. J. Laser-induced Hole Filling and Spectral Diffusion of Aluminum Phthalocyanine Tetrasulfonate in Hyperquenched Glassy Films, J. Chem. Phys. 1999, $110,4820-4827$.

19. Grozdanov, D.; Herascu, N.; Reinot, T.; Jankowiak, R.; Zazubovich, V. Low-Temperature Protein Dynamics of the B800 Molecules in the LH2 Light-Harvesting Complex: Spectral Hole Burning Study and Comparison with Single Photosynthetic Complex Spectroscopy, J. Phys. Chem. B 2010, 114, 3426-3438.

20. Love, S. P.; Mungan, C. E.; Sievers A. J.; Campbell, J. A. Persistent Infrared Spectral Hole Burning of $\mathrm{Tb}^{3+}$ in the Glasslike Mixed Crystal Ba1-X-YLax $\mathrm{Tb}_{\mathrm{y}} \mathrm{F}_{2+\mathrm{x}+\mathrm{y}}$, J. Opt. Soc. Am. B 1992, 9, 794-799.

21. Dang, N. C.; Reinot, T.; Reppert, M.; Jankowiak, R. Temperature Dependence of Hole Growth Kinetics in Aluminum-Phthalocyanine-Tetrasulfonate in Hyperquenched Glassy Water, J. Phys. Chem. B 2007, 111, 1582-1589.

22. Oikawa, H.; Fujiyoshi, S.; Dewa, T.; Nango, M. Matsushita, M. How Deep is the Potential Well Confining a Protein in a Specific Conformation? A Single-molecule Study on Temperature Dependence of Conformational Change between 5 and $18 \mathrm{~K}, \mathrm{~J}$. Am. Chem. Soc. 2008, 130, 4580-4581.

23. Adolphs, J.; Berrer, M.; Renger, T. Hole-Burning Spectroscopy on Excitonically Coupled Pigments in Proteins: Theory Meets Experiment, J. Am. Chem. Soc. 2016, 138, 2993-3001.

24. Loll, B.; Kern, J.; Saenger, W.; Zouni, A.; Biesiadka, J. Towards Complete Cofactor Arrangement in the 3.0 A Resolution Structure of Photosystem II, Nature 2005, 438, 10401044. 
25. Yamashita, E.; Zhang, H.; Cramer, W.A. Structure of the Cytochrome $b_{6} f$ complex: Quinone Analogue Inhibitors as Ligands of Heme cn, J. Mol. Biol. 2007, 370, 39-52.

26. Heuer, A.; Neu, P. Tunneling Dynamics of Side Chains and Defects in Proteins, Polymer Glasses, and OH-doped Network Glasses, J. Chem. Phys. 1997, 107, 8686-8696.

27. Fenimore, P. W.; Frauenfelder, H.; McMahon, B. H.; Parak, F. G. Slaving: Solvent Fluctuations Dominate Protein Dynamics and Functions, Proc. Natl. Acad. Sci. USA 2003, 99, 16047-16051.

28. Kim, W.-H.; Reinot, T.; Hayes, J.M.; Small, G.J. Nonphotochemical Hole Burning in Hyperquenched Glassy Films of Water: A Pronounced Deuteration Effect, J. Chem. Phys. 1996, 104, 6415-6417.

29. Monks-Corrigan, T.; Riesen, H. Host Deuteration Effects in Non-photochemical Spectral Hole-burning in the $\mathrm{R}_{1}$-line of $\left[\mathrm{Cr}(\text { oxalate })_{3}\right]^{3-}$ in Ethylene Glycol/water, Chem. Phys. Lett. 2006, 419, 321-325.

30. Riesen, H.; Hughes, J. L. Massive Enhancement of Persistent Spectral Hole-burning in the R-Lines of $\mathrm{NaMgAl}\left(\right.$ oxalate) $3^{\cdot}$ 9H2O:Cr(III) by Partial Deuteration, Chem. Phys. Lett. 2003, $372,563-568$.

31. Lujan, M.A.; Lorente, P.; Zazubovich, V.; Picorel, R. A simple and efficient method to prepare pure dimers and monomers of the cytochrome $b_{6} f$ complex from spinach. Photosynth. Res. 2017, 132, 305-305.

32. Baniulis, D.; Zhang, H.; Zakharova, T.; Hassan, S.S.; Cramer, W.A. Purification and crystallization of the cyanobacterial cytochrome $b_{6} f$ complex. In Photosynthesis Research Protocols, Methods in Molecular Biology, Vol. 684 (R. Carpentier, Ed.), 2011, 65-77. 
33. Pierre, Y.; Breyton, C.; Kramer, D.; Popot, J. L. Purification and Characterization of the Cytochrome b 6 f Complex from Chlamydomonas reinhardtii, J. Biol. Chem. 1995, 270, 29342-29349.

34. Peterman, E. J.; Wenk, S.-O.; Pullerits, T.; Pålsson, L.-O.; van Grondelle, R.; Dekker, J. P.; Rogner, M.; van Amerongen, H. Fluorescence and Absorption Spectroscopy of the Weakly Fluorescent Chlorophyll $a$ in Cytochrome b ${ }_{6}$ f of Synechocystis PCC6803, Biophys. J. 1998, 75, 389-398.

35. Dashdorj, N.; Zhang, H.; Kim, H.; Yan, J.; Cramer, W. A.; Savikhin, S. The Single Chlorophyll $a$ Molecule in the Cytochrome $b_{6} f$ Complex: Unusual Optical Properties Protect the Complex against Singlet Oxygen, Biophys. J. 2005, 88, 4178-4187.

36. Yan, J.; Dashdorj, N.; Baniulis, D.; Yamashita, E.; Savikhin, S.; Cramer, W. A. On the Structural Role of the Aromatic Residue Environment of the Chlorophyll $a$ in the Cytochrome b 6 f Complex, Biochemistry 2008, 47, 3654-3661.

37. Herascu, N.; Ahmouda, S.; Picorel, R.; Seibert, M.; Jankowiak, R.; Zazubovich, V. Effects of the Distributions of Energy or Charge Transfer Rates on Spectral Hole Burning in Pigment-Protein Complexes at Low Temperatures, J. Phys. Chem. B 2011, 115, 1509815109.

38. Agostiano, A.; Della Monica, M.; Palazzo G.; Trotta, M. Chlorophyll $a$ Autoaggregation in Water Rich Region, Biophys. Chem. 1993, 47, 193-202.

39. Vladkova, R. Chlorophyll $a$ Self-assembly in Polar Solvent-Water Mixtures, Photochem. Photobiol. 2000, 71, 71-83. 
40. Byrdin, M.; Jordan, P.; Krauß, N.; Fromme, P.; Stehlik, D.; Schlodder, E. Light Harvesting in Photosystem I: Modeling Based on the 2.5- $\AA$ Structure of Photosystem I from Synechococcus elongatus, Biophys. J. 2002, 83, 433-457.

41. Hughes, J. L.; Krausz, E.; Smith, P. J.; Pace, R. J.; Riesen, H. Probing the lowest energy chlorophyll a states of photosystem II via selective spectroscopy: new insights on P680. Photosynth Res. 2005, 84, 93-98.

42. Hughes, J. L.; Picorel, R.; Seibert, M.; Krausz, E. Photophysical Behavior and Assignment of the Low-Energy Chlorophyll States in the CP43 Proximal Antenna Protein of Higher Plant Photosystem II, Biochemistry 2006, 45, 12345-12357

43. Hughes, J. L.; Prince, B. J.; Peterson Årsköld, S., Krausz, E.; Pace, R. J.; Picorel, R.; Seibert, M. Photo-conversion of chlorophylls in higher-plant CP43 characterized by persistent spectral hole burning at 1.7K, J. Lumin. 2004, 108, 131-136.

44. Najafi, M.; Zazubovich, V. Monte-Carlo Modeling of Spectral Diffusion Employing Multiwell Protein Energy Landscapes: Application to Pigment-Protein Complexes Involved in Photosynthesis, J. Phys. Chem. B 2015, 119, 7911-7921.

45. Krivchikov, A. I.; Bermejo, F. J.; Sharapova, I. V.; Korolyuk, O. A.; Romantsova, O. O. Deuteration Effects in the Thermal Conductivity of Molecular Glasses, Low Temp. Phys. 2011, 37, 517-523.

46. Talon, C.; Zou, Q. W.; Ramos, M. A.; Villar, R.; Vieira, S. Low-temperature Specific Heat and Thermal Conductivity of Glycerol, Phys. Rev. B 2001, 65, 012203.

47. Ahmad, N. Thermal Conductivity of Ice, Phys. Stat. Sol. (b) 1994, 181, 37-44. 
48. Choi, J.; Bischof, J. C. Review of Biomaterial Thermal Property Measurements in the Cryogenic Regime and Their Use for Prediction of Equilibrium and Non-equilibrium Freezing Applications in Cryobiology, Cryobiology, 2010, 60, 52-70.

49. Yu. X.; Leitner, D. M. Heat flow in Proteins: Computation of Thermal Transport Coefficients, J. Chem. Phys. 2005, 122, 054902.

50. Lervik, A.; Bresme, F.; Kjelstrup, S.; Bedeaux, D.; Rubi, J. M. Heat Transfer in Protein-Water Interfaces, Phys. Chem. Chem. Phys., 2010, 12, 1610-1617.

51. Shi, L.; Wu, G.; Wang, H.-L.; Yu, X.-M. Interfacial Thermal Contact Resistance between Aluminum Nitride and Copper at Cryogenic Temperature, Heat Mass Transfer 2012, 48, 999-1004,

52. Pohorille, A.; Pratt, L. R.; LaViolette, R. A.; Wilson, M. A.; MacElroy, R. D. Comparison of the Structure of Harmonic Aqueous Glasses and Liquid Water, J. Chem. Phys. 1988, 87, 6070-6077;

53. Cho, M.; Fleming, G. R.; Saito, S.; Ohmine, I.; Stratt, R. M. Instantaneous Normal Mode Analysis of Liquid Water, J. Chem. Phys. 1994, 100, 6672-6683;

54. Go, N.; Noguti, T.; Nishikawa, T. Dynamics of a Small Globular Protein in Terms of Lowfrequency Vibrational Modes, Proc. Natl. Acad. Sci. USA 1983, 80, 3696-3700.

55. Brooks, B. R.; Karplus, M. Harmonic Dynamics of Proteins: Normal Modes and Fluctuations in Bovine Pancreatic Trypsin Inhibitor, Proc. Natl. Acad. Sci. USA 1983, 80, $6571-6575$.

56. Levitt, M.; Sander, C.; Stern, P. S. Protein Normal-mode Dynamics: Trypsin Inhibitor, Crambin, Ribonuclease and Lysozyme, J. Mol. Biol. 1985, 181, 423-447. 
TOC Graphic

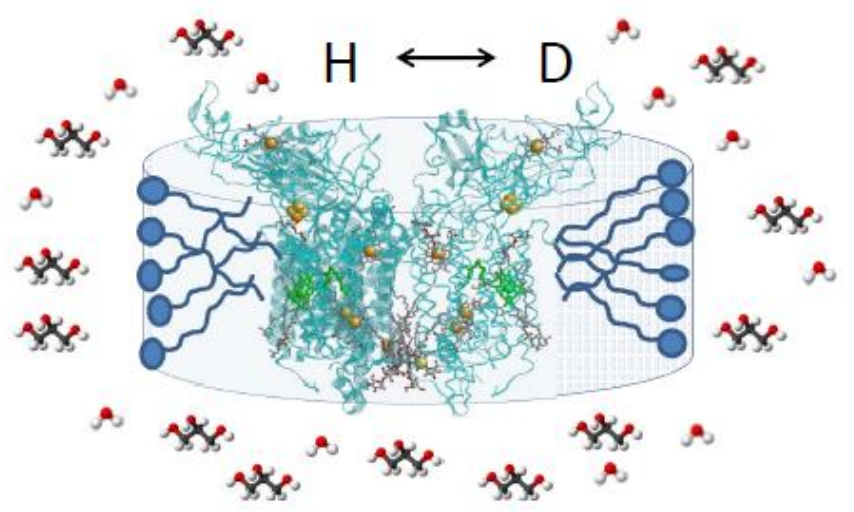

